\title{
Effectiveness of Intermittent Preventive Treatment in Pregnancy with Sulphadoxine-Pyrimethamine against Submicroscopic falciparum Malaria in Central Region, Ghana
}

\author{
Ekene K. Nwaefuna, ${ }^{1}$ Richmond Afoakwah, ${ }^{2}$ Verner N. Orish, ${ }^{3}$ \\ Alexander Egyir-Yawson, ${ }^{1}$ and Johnson N. Boampong ${ }^{2}$ \\ ${ }^{1}$ Vector Genetics Laboratory, Biotechnology and Nuclear Agriculture Research Institute, \\ Ghana Atomic Energy Commission, Accra, Ghana \\ ${ }^{2}$ Department of Biomedical and Forensic Sciences, University of Cape Coast, Cape Coast, Ghana \\ ${ }^{3}$ Department of Internal Medicine, Effia-Nkwanta Regional Hospital Sekondi-Takoradi, \\ Sekondi, Western Region, Ghana
}

Correspondence should be addressed to Ekene K. Nwaefuna; nwaekene@live.com

Received 28 May 2015; Revised 21 August 2015; Accepted 30 August 2015

Academic Editor: Xin-zhuan Su

Copyright (C) 2015 Ekene K. Nwaefuna et al. This is an open access article distributed under the Creative Commons Attribution License, which permits unrestricted use, distribution, and reproduction in any medium, provided the original work is properly cited.

\begin{abstract}
Malaria infections undetectable by microscopy but detectable by Polymerase Chain Reaction (PCR) (submicroscopic malaria) are common in endemic areas like Ghana. Submicroscopic malaria has been linked with severe pregnancy outcomes as well as contributing to malaria transmission. In this cross-sectional study 872 consenting pregnant women (gestation $\geq 20$ weeks) were recruited from 8 hospitals in Central Region, Ghana, between July and December 2009. Malaria infection was detected by microscopy and PCR. Haemoglobin was measured and anaemia was defined as haemoglobin lower than $11 \mathrm{~g} / \mathrm{dL}$. Majority of the women, 555 (63.6\%), were Intermittent Preventive Treatment in Pregnancy with Sulphadoxine-Pyrimethamine (IPTp-SP) users while 234 (36.4\%) were nonusers. The prevalence of malaria by microscopy was 20.9\% (182/872) and 9.7\% (67/688) of microscopy negative women had submicroscopic malaria. IPTp-SP usage significantly (odds ratio $=0.13,95 \%$ confidence interval $=0.07-0.23$, $p=0.005)$ reduced the prevalence of submicroscopic malaria as more nonusers (51/234) than users (16/454) were PCR positive. After controlling for other variables the effect of IPTp-SP remained statistically significant (odds ratio $=0.11,95 \%$ confidence interval $=0.02-0.22, p=0.006)$. These results suggest that Intermittent Preventive Treatment with Sulphadoxine-Pyrimethamine is useful in the reduction of submicroscopic malaria in pregnancy.
\end{abstract}

\section{Introduction}

Malaria accounts for the highest cause of mortality in Africa with $90 \%$ of the global malaria deaths occurring on this continent. It constitutes $9 \%$ of the disease burden to the people of Africa and is responsible for $25 \%$ of deaths below the age of five years [1]. Susceptibility to malaria is increased by up to 3-fold during pregnancy, thereby making pregnant women one of the most vulnerable groups [2].

Submicroscopic malaria, defined as plasmodium infection below the threshold detectable by microscopy but detectable by Polymerase Chain Reaction (PCR), has received lots of attention due to its role in disease transmission [3]. Due to the sequestration of Plasmodium falciparum infected red blood cells in the placenta of pregnant women, malaria diagnosisfrom peripheral blood has been inadequate. However PCR diagnosis is effective in detecting not only parasitaemia below the threshold of microscopy but also placental infections [4]. PCR has been reported to be more sensitive in the detection of malaria during pregnancy than the microscopy of placenta impression smears; $65 \%$ of women negative for malaria by microscopy were positive by PCR [5]. 
In areas of stable malaria transmission in Africa, the WHO recommends a package of Intermittent Preventive Treatment in Pregnancy with Sulphadoxine-Pyrimethamine (IPTp-SP) and use of Insecticide Treated Nets. Nonetheless, achievement of high coverage of these preventive interventions among pregnant women remains elusive for many countries in Sub-Saharan Africa [1]. In Ghana, the WHO recommendations were adopted and finally implemented in 2005; however coverage remains generally low. Nonetheless, several studies have continued to report its effectiveness in prevention of pregnancy associated malaria [6-8]. It, however, becomes necessary to continue to access its usefulness in varying dynamics of pregnancy associated malaria. In this study, we investigated the effectiveness of IPTp-SP against submicroscopic malaria among pregnant women in Central Region, Ghana, barring all constraints associated with the administration of this intervention.

\section{Materials and Methods}

2.1. Study Area and Population. The study area has been described elsewhere [9], but briefly the study was carried out in Central Region, Ghana. Central Region $\left(5^{\circ} 30^{\prime} 0^{\prime \prime} \mathrm{N}\right.$, $1^{\circ} 0^{\prime} 0^{\prime \prime} \mathrm{W}$ ) occupies an area of 9,826 square kilometers, which is about $6.6 \%$ of the land area of Ghana. It has an estimated population of $1,805,488$ and an annual population growth rate of $2.1 \%$ with 17 administrative districts [10]. In 2008, the region registered 86,971 pregnant women thus recording $115.5 \%$ antenatal care coverage and $56.3 \%$ supervised delivery [11].

2.2. Ethical Clearance. Ethical approval was sought from the Ghana Health Service Ethical Review Committee (ID number: GHS - ERC - 13/7/09). All activities including sample collection, processing, and analysis were carried out as required by the committee. The Central Regional Health Directorate also approved the protocols used in this study.

2.3. Study Design. This research was a cross-sectional study. The sample sites (hospitals) were purposefully selected while the participants randomly selected. The region was divided into two belts and four hospitals were selected from each belt: the Coastal Belt ( $15 \mathrm{~km}$ inland) (Moree Health Centre, Kasoa Health Centre, Elmina Health Centre, and Cape Coast Metropolitan Hospital) and the Forest Belt (Swedru Hospital, Our Lady of Grace Hospital Asikuma, Saint Francis Xavier Hospital, Assin Foso, and Dunkwa-on-Offin District Hospital). The study was designed to evaluate the effectiveness of IPTp-SP in the normal state of its implementation barring all challenges and obstacles as a result of health system problems. However, only hospitals with functional ANC clinics where the IPTp-SP policy is implemented were included in the study.

2.4. Sample Size Determination. Using the method described by Fisher a sample size of seven hundred and ten (710) was required to represent the population of pregnant women in Central Region based on the following assumptions: standard normal deviation of 1.96 corresponding to $95 \%$ confidence interval, 29\% prevalence of malaria in pregnancy, and 0.05 degrees of accuracy [12]. The total number of pregnant women reporting to each health facility yearly was made as a fraction of the total number of pregnant women seen in all these health facilities annually. The proportion for each facility was used to determine the number of pregnant women sampled from that facility. However eight hundred and seventy-two (872) samples were collected.

2.5. Selection Criteria. The pregnant women were recruited from antenatal care units of the hospitals after written informed consent was obtained according to the Ethical Review Committee of the Ghana Health Service. Only pregnant women $\geq 20$ weeks (second or third trimester) were recruited. Pregnant women with complications such as sepsis and hemorrhage were excluded.

2.6. Data Collection. Eight hundred and seventy-two [872] pregnant women participated in the study. Some health and obstetric pieces of information such as gestation, gravidity, Glucose-6-Phosphate Dehydrogenase (G6PD) status, and SP intake were obtained from the Antenatal Clinic (ANC) booklets of participants; other information was collected using a structured questionnaire administered by trained research assistants. The assistants were recruited and trained to explain the questionnaire in their own local languages. Three milliliters $(3 \mathrm{~mL})$ of venous blood was collected from the median cubital or cephalic veins of the arm of pregnant women into labeled vacutainers, containing Ethelene Diamine Tetra Acetic Acid (EDTA). One hundred microliters $(100 \mu \mathrm{L})$ of the blood was spotted on Whatman No. 1 filter papers, air dried and placed in labeled plastic envelopes, and then stored at $-20^{\circ} \mathrm{C}$ until usage. Thick blood films were prepared on labeled microscope slides, Giemsa-stained and observed by a trained and licensed senior biomedical scientist and a principal biomedical scientist for malaria diagnosis. A sample was labelled positive if one or more parasites are present and negative if none is seen after observing 100 high power fields. Haemoglobin $(\mathrm{Hb})$ level was determined using the SYSMEX KX - 21 haematology analyser (Sysmex Corporation, Kobe, Japan) and the numerical values were recorded. The sodium metabisulphite method was used to determine sickling status as described by Cheesbrough [13].

2.7. Molecular Analysis. Plasmodium falciparum DNA was extracted from blood spots on filter paper using the CHELEX Method of extraction as described by Bereczky et al. [14]. Extracted DNA samples were stored at $-20^{\circ} \mathrm{C}$ until usage. Of the 690 participants who were malaria negative by microscopy and included in the molecular analysis, only 688 were successful. A nested Polymerase Chain Reaction (PCR) was carried out following the method described by Beck and Ley [15] with slight modifications. The primary PCR amplified a 642 bp Plasmodium falciparum dihydrofolate reductase ( $p f d h f r)$ gene. The reaction was set up in a 96-well plate to a final volume of $25 \mu \mathrm{L}$ per well containing $1 \mathrm{x}$ buffer, $1.5 \mathrm{mM} \mathrm{MgCl} 2,200 \mu \mathrm{M}$ dNTP mix, and $0.25 \mu \mathrm{M}$ of each primer $\left(\mathrm{M}_{1}=5^{\prime}\right.$ TTT ATG ATG GAA CAA GTC TGC 3', 


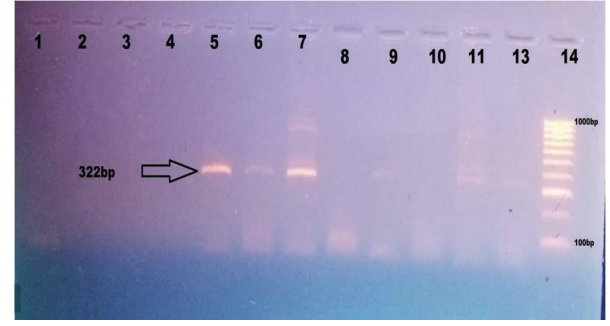

Figure 1: Gel photograph of the 2nd amplification, showing the $322 \mathrm{bp}$ product. Lane $1=$ negative control, lane $5=$ positive control, and lanes $2-4$ and $6-13=$ participants' samples.

$\mathrm{M}_{5}=5^{\prime}$ AGT ATA TAC ATC GCT AAC AGA $3^{\prime}$ ). One denaturing step at $94^{\circ} \mathrm{C}$ for 3 minutes was followed by 40 amplification cycles. Each cycle constituted a denaturation step at $94^{\circ} \mathrm{C}$ for 1 minute, an annealing step at $50^{\circ} \mathrm{C}$ for 2 minutes, and an extension step at $72^{\circ} \mathrm{C}$ for 2 minutes. This was followed by a final extension step $72^{\circ} \mathrm{C}$ for 10 minutes. This cycler condition lasted for 4 hours 30 minutes. Five microlitres of the primary PCR product was used for the second amplification using the following primers: $\left(\mathrm{F}^{\prime}=5\right.$ GAA ATG ATG GAA CAA GTC TGC GAC GTT $3^{\prime}$ ) and $\left(2 \mathrm{~F}=5^{\prime}\right.$ TTA ATT TCC CAA GTA AAA CTA TTA GAG CTT3 $^{\prime}$ ) corresponding to codon 59-108 of the pfdhfr gene. The reaction was set up in a 96-well plate to a final volume of $25 \mu \mathrm{L}$ per well containing $1 \mathrm{x}$ buffer, $1.5 \mathrm{mM} \mathrm{MgCl}_{2}, 200 \mu \mathrm{M}$ dNTP mix, and $0.25 \mu \mathrm{M}$ of each primer. One denaturing step at $94^{\circ} \mathrm{C}$ for 2 minutes was followed by 40 amplification cycles. Each cycle constituted a denaturation step at $94^{\circ} \mathrm{C}$ for 1 minute, an annealing step at $45^{\circ} \mathrm{C}$ for 1 minute, and an extension step at $72^{\circ} \mathrm{C}$ for 2 minutes. This was followed by a final extension step $72^{\circ} \mathrm{C}$ for 10 minutes. This cycler condition lasted for 3 hours 30 minutes. Eight microlitres $(8 \mu \mathrm{L})$ of each nested PCR product was separated on 2\% agarose gel stained with ethidium bromide. The gels were then photographed (Figure 1) under UV light. Any sample with a nested PCR product that showed a band equivalent to the $322 \mathrm{bp}$ mark on the molecular weight marker was labeled as positive for malaria, specifically Plasmodium falciparum infection.

2.8. Definitions. IPTp-SP users are pregnant women who have taken at least one dose of SP and no other malaria prophylaxis while nonusers have not taken SP during the present pregnancy at the time of the study. Gravidity was grouped as Primigravidae (1st pregnancy), Secundigravida (2nd pregnancy), and multigravidae (3rd or more pregnancies). A malaria positive blood smear was regarded as microscopy positive. A microscopy negative but PCR positive sample was regarded as submicroscopic malaria. A participant was anaemic if $\mathrm{Hb}<11.0 \mathrm{~g} / \mathrm{dL}$.

2.9. Statistical Analysis. All data was entered into SPSS statistical software version 17.0 for Windows and analyzed. Frequencies and Pearson's chi-square were used to compare relationships between variables. To determine the independent effect of IPTp-SP on submicroscopic malaria, logistic
TABLE 1: Background characteristics of participating women.

\begin{tabular}{lccc}
\hline Variables & $\begin{array}{c}\text { IPIp-SP } \\
(n=555)\end{array}$ & $\begin{array}{c}\text { No IPTp-SP } \\
(n=317)\end{array}$ & $p$ \\
\hline Mean age \pm SD & $33.4 \pm 4.9$ & $32.1 \pm 5.1$ & 0.33 \\
$\quad$ Range & $20-46$ & $17-44$ & \\
Educational level & & & 0.011 \\
$\quad$ None & $93(16.8 \%)$ & $59(18.6 \%)$ & \\
Primary & $100(18.0 \%)$ & $39(12.4 \%)$ & \\
Secondary & $336(60.5 \%)$ & $189(59.5 \%)$ & \\
$\quad$ Tertiary & $26(4.6 \%)$ & $30(9.5 \%)$ & \\
Occupation & & & 0.001 \\
$\quad$ Unemployed & $93(16.8 \%)$ & $46(14.7 \%)$ & \\
$\quad$ Unskilled & $299(54.0 \%)$ & $145(46.0 \%)$ & \\
Para-profession & $145(26.1 \%)$ & $95(30.0 \%)$ & \\
$\quad$ White collar & $10(1.8 \%)$ & $9(2.8 \%)$ & \\
$\quad$ Student & $8(1.4 \%)$ & $22(6.9 \%)$ & \\
\hline
\end{tabular}

regression modeling was applied. Possible confounding factors such as gestation, occupation, gravidity, G6PD, and sickling status were controlled. Odds ratio (OR) and confidence interval (CI) were calculated by using logistic regression in multivariate analyses. In the logistic regression analysis, all variables were treated as categorical except gestation and gravidity that were treated as categorical. For all statistical tests, $p<0.05$ was considered significant.

\section{Results}

3.1. General Characteristics of Participants. A total of 872 pregnant women consented to participate in the study. The mean age of the participants was 32.9 years ranging from 17 to 46 years. When grouped by IPTp-SP usage, there was no significant difference $(p=0.33)$ in the mean age for users 33.4 years range (20-46) and nonusers $32.1(17-44)$. Women using IPTp-SP were significantly more educated $(p=0.011)$ and better employed $(p=0.001)$ than nonusers (Table 1$)$.

3.2. Health and Obstetric Information of Participants. There was a significant difference between the IPTp-SP users and nonusers based on trimester $(p=0.001)$ and gravidity $(p=$ 0.023 ) as more women with pregnancy experience were users than nonusers. There was no association between the two groups in terms of G6PD ( $p=0.719)$ and sickling status $(p=0.123)($ Table 2$)$.

3.3. Submicroscopic Malaria in Pregnancy. The prevalence of submicroscopic malaria was not significantly affected by the gestation $(p=0.575)$ and gravidity $(p=0.665)$. More than half of the women with submicroscopic malaria were anaemic. Submicroscopic malaria significantly resulted in anaemia $(p=0.003)$ (Table 3$)$.

Increasing dosage of SP had a significant $(p=0.018)$ preventive effect on submicroscopic malaria. The prevalence of malaria by microscopy was $20.9 \%(182 / 872)$ and by PCR 
TABLE 2: Health and obstetric information of participants.

\begin{tabular}{lccc}
\hline Variables & $\begin{array}{c}\text { IPIp-SP } \\
(n=555)\end{array}$ & $\begin{array}{c}\text { No IPTp-SP } \\
(n=317)\end{array}$ & $p$ \\
\hline $\begin{array}{l}\text { Trimester } \\
\text { Second }\end{array}$ & $397(71.5 \%)$ & $285(89.9 \%)$ & 0.0001 \\
$\quad$ Third & $158(28.5 \%)$ & $32(10.1 \%)$ & \\
Gravidity & & & 0.023 \\
$\quad$ Primigravidae & $146(26.3 \%)$ & $111(35.0 \%)$ & \\
$\quad$ Secundigravida & $167(30.1 \%)$ & $88(27.8 \%)$ & \\
$\quad$ Multigravidae & $242(43.6 \%)$ & $118(37.2 \%)$ & \\
G6PD & & $314(99.1 \%)$ & \\
$\quad$ No defect & $551(99.3 \%)$ & $3(0.09 \%)$ & \\
$\quad$ Partial defect & $4(0.07 \%)$ & & 0.123 \\
Sickling status & & $17(5.4 \%)$ & \\
$\quad$ Positive & $61(11.0 \%)$ & $300(94.6 \%)$ & \\
$\quad$ Negative & $494(89.0 \%)$ & & \\
& &
\end{tabular}

TABLE 3: Submicroscopic malaria dynamics among pregnant women in Ghana.

\begin{tabular}{lccc}
\hline \multirow{2}{*}{ Variables } & \multicolumn{2}{c}{ Submicroscopic malaria } & \multirow{2}{*}{$p$} \\
& Positive $(n=67)$ & Negative $(n=621)$ & 0.575 \\
\hline Trimester & $51(76.1 \%)$ & $491(79.1 \%)$ & \\
Second & $16(23.9 \%)$ & $130(20.9 \%)$ & \\
Third & & & 0.665 \\
Gravidity & $16(23.9 \%)$ & $172(27.7 \%)$ & \\
Primigravidae & $23(34.3 \%)$ & $183(29.5 \%)$ & \\
Secundigravida & $28(41.8 \%)$ & $266(42.8 \%)$ & \\
Multigravidae & & & 0.003 \\
Anaemia & $35(52.2 \%)$ & $210(33.8 \%)$ & \\
Anaemic & $32(47.8 \%)$ & $411(66.2 \%)$ & \\
Normal & & & 0.018 \\
SP dosage & $16(23.9 \%)$ & $218(35.1 \%)$ & \\
None & $36(53.7 \%)$ & $293(47.2 \%)$ & \\
Once & $14(20.9 \%)$ & $86(13.8 \%)$ & \\
Twice & $1(1.5 \%)$ & $24(3.9 \%)$ & \\
Thrice & &
\end{tabular}

(submicroscopic) was 9.7\% (67/688) but when submicroscopic infection was added it shot up to $28.6 \%$ (249/872). IPTp-SP usage significantly reduced submicroscopic falciparum infection $(\mathrm{OR}=0.13,95 \% \mathrm{CI}=0.07-0.23, p=0.005)$ (Table 4).

From the logistic regression, after controlling for possible confounders, the effect of IPTp-SP and gestation on submicroscopic malaria remained statistically significant $(\mathrm{OR}=$ $0.11,95 \% \mathrm{CI}=0.02-0.22, p=0.006$; OR $=0.28,95 \% \mathrm{CI}=$ $0.18-0.42, p=0.001$ ) (Table 5).

\section{Discussion}

Eight hundred and seventy-two (872) consenting pregnant women participated in the study. All the participants were eligible to use SP but only 555/872 (63.6\%) had used IPTp. This may be because the women report late for antenatal care or they do not visit regularly. Though data on ANC attendance was not collected in the present study, late first ANC clinic enrolments and fewer visits have been reported to limit IPTpSP usage [16]. IPTp-SP users were not significantly $(p=0.33)$ older than nonusers but weresignificantly more educated and better employed than nonusers. This should buttress the importance of education in health care; as such health education schemes for pregnant women should be intensified in rural areas. The social status of Ghanaians has often been linked to health care as people with stable income have been shown to exhibit better health seeking behavior [17].

More women with pregnancy experience were IPTp users; this might be because the women were already conversant with the routine procedures at the ANC. Thus, they reported early and regularly. More third trimester participants were IPTp users as there was a significant association between gestation and IPTp usage. This is not surprising because at the third trimester pregnant women are expected to have had several ANC visits during which the IPTp intervention should have been administered. G6PD deficiency and sickling status did not vary significantly between the two groups.

The prevalence of malaria by microscopy was $20.9 \%$ $(182 / 872)$ while that of submicroscopic malaria was $9.7 \%$ (67/688). Submicroscopic malaria in pregnancy may be as a result of parasitaemia below the threshold of microscopy or the sequestration of infected red blood cells in the placenta [18]. These results are different from findings of a similar study in Kumasi, Ghana, where prevalence by microscopy was $51 \%$, PCR was $49 \%$, and both were $63 \%$ [19]. Despite the fact that both studies were carried out in different geographical areas of Ghana, the Kumasi study was conducted prior to the implementation of IPTp-SP in Ghana; thus the difference in prevalence may be as a result of the implementation of malaria control measures.

A number of studies have suggested that malaria infection of any parasite density may have a harmful effect on pregnant women and their developing fetuses [20,21]. Submicroscopic malaria has been highly implicated for its contribution to malariatransmission as well as complicating control programmes and treatment [22]. In a recent study carried out in the Sudan, pregnant women with submicroscopic malaria had a higher risk of having low birth weight babies [23]. Since malaria elimination programs have been directed towards mass screening and treatment of asymptomatic individuals, further research should strive to elucidate the degree to which submicroscopic malaria contributes to the infectious reservoir and, in turn, what diagnostic detection threshold is needed to effectively interrupt transmission [3].

The prevalence of submicroscopic malaria did not differ significantly by gestation and gravidity $(p=0.575$ and $0.665)$, respectively. This is contrary to an earlier study that linked higher submicroscopic infections to primigravidae [19]. However after controlling for possible confounding factors the prevalence of submicroscopic malaria became significant by gestational age $(p=0.001)$. More than half (52\%) of the participants with submicroscopic malaria were anaemic $(\mathrm{Hb}<11.0 \mathrm{~g} / \mathrm{dL})$. Anaemia in pregnancy is however multifactorial and can be caused by a number of reasons 
TABLE 4: Effect of IPTp-SP on submicroscopic malaria in pregnancy.

(a)

\begin{tabular}{lcccc}
\hline Variable & $\begin{array}{c}\text { IPIp-SP } \\
(n=454)\end{array}$ & $\begin{array}{c}\text { No IPTp-SP } \\
(n=234)\end{array}$ & Odds ratio & $95 \%$ CI \\
\hline $\begin{array}{l}\text { Submicroscopic } \\
\text { Positive }\end{array}$ & $16(3.5 \%)$ & $51(21.8 \%)$ & 0.13 & $0.07-0.23$ \\
$\quad$ Negative & $438(96.5 \%)$ & $183(79.2 \%)$ & & 0.005 \\
\hline
\end{tabular}

(b)

\begin{tabular}{|c|c|c|c|c|c|}
\hline Variables & $\begin{array}{l}\text { IPIp-SP } \\
(n=555)\end{array}$ & $\begin{array}{c}\text { No IPTp-SP } \\
\quad(n=317)\end{array}$ & Odds ratio & $95 \% \mathrm{CI}$ & $p$ \\
\hline Microscopic & & & 0.08 & $0.05-0.13$ & 0.002 \\
\hline Positive & $38(6.8 \%)$ & $144(45.4 \%)$ & & & \\
\hline Negative & $517(93.2 \%)$ & $173(54.6 \%)$ & & & \\
\hline Microscopic + submicroscopic & & & 0.06 & $0.04-0.09$ & 0.001 \\
\hline Positive & $54(9.7 \%)$ & $195(61.5 \%)$ & & & \\
\hline Negative & $551(90.3 \%)$ & $139(39.5 \%)$ & & & \\
\hline
\end{tabular}

TABLE 5: Logistic regression analyses of confounders of submicroscopic malaria infection among pregnant women from selected health facilities in Central Region, Ghana.

\begin{tabular}{lccc}
\hline Variable & Odds ratio & $95 \%$ CI & $p$ \\
\hline Education & 1.02 & $0.84-1.09$ & 0.721 \\
Gravidity & 0.74 & $0.31-1.73$ & 0.092 \\
Occupation & 1.55 & $0.42-4.40$ & 0.041 \\
Gestation & 0.28 & $0.18-0.42$ & 0.001 \\
Sickle cell positive & 2.18 & $1.24-3.80$ & 0.061 \\
IPTp-SP & 0.11 & $0.02-0.22$ & 0.006 \\
G6PD partial defect & 1.75 & $0.44-7.06$ & 0.428 \\
\hline
\end{tabular}

including poor nutrition, intestinal parasites, and socioeconomic status which have not been measured in this study. Nonetheless, similar studies have reported the contribution of malaria to anaemia in pregnancy $[6,24]$. This is indicative of the effect of submicroscopic $P$. falciparum infection in pregnancy. These submicroscopic infections may be as a result of prevailing placenta infection, because diagnosis of placenta malaria from peripheral blood by PCR has been reported to approach $100 \%$ accuracy [25].

Due to the profound consequences of submicroscopic malaria, it is very important that the IPTp intervention should not only be effective in clearing parasite from the peripheral blood but also prevent submicroscopic and placental infections. Usage of IPTp significantly reduces the presence of malaria infection both at microscopic and submicroscopic levels ( $p=0.002, p=0.005$, resp.). Studies have reported the protective efficacy of Sulphadoxine-Pyrimethamine when used as malaria prophylaxis during pregnancy. We further indicate here that its efficacy extends beyond the threshold of microscopic to submicroscopic infections. Recent studies have reported treatment failures as well as rise in the prevalence of molecular markers of SP resistance. In Ghana, for example, prevalence of molecular markers of
SP resistance was 73\%; nevertheless SP remains effective in preventing Ghanaian women from malaria [6-8]. It becomes important for further research to be carried out to clarify the relationships between drug resistance, treatment failure, and molecular markers of SP resistance. The prevalence of submicroscopic malaria reduced significantly $(p=0.018)$ with increasing dosage of SP. One clinical trial in Malawi also underscored the increased efficacy of SP with increasing doses among delivering women [26].

The major limitation of this study is the absence of data on pregnancy outcomes such as placental malaria, congenital and perinatal mortality, and birth weight. This was due to the cross-sectional nature of the study and did not allow for participants to be followed till delivery. However, reports have been made on the useful effect of IPTp-SP in the reduction of low birth weight (LBW), neonatal mortality (NM), and placental malaria (PM) $[8,24,25,27]$. Another limiting factor may be the general health systems barriers, which include staff motivation, lack of skilled personnel, and availability of medications, capable of hindering the success of health care policies in Sub-Saharan Africa [28, 29]. However, only hospitals with vibrant ANC clinics were selected for this study: an attempt to limit the effect of this limitation.

Intermittent Preventive Treatment in Pregnancy with Sulphadoxine-Pyrimethamine continues to be a useful regimen for the prevention of pregnancy associated malaria and submicroscopic malaria in pregnancy.

\section{Conclusion}

IPTp-SP is useful in the prevention of pregnancy associated malaria in Ghana.

\section{Conflict of Interests}

The authors declare that there is no conflict of interests regarding the publication of this paper. 


\section{Acknowledgments}

The authors are grateful to all the staff of the ANC and the hospitals they visited for sampling. Special thanks go to the participants who consented to participate in the study as well as technicians at the Vector Genetics Laboratory at Ghana Atomic Energy Commission, Accra, Ghana.

\section{References}

[1] WHO, The World Malaria Report, World Health Organization, Geneva, Switzerland, 2009.

[2] E. Sappenfield, D. J. Jamieson, and A. P. Kourtis, "Pregnancy and susceptibility to infectious diseases," Infectious Diseases in Obstetrics and Gynecology, vol. 2013, Article ID 752852, 8 pages, 2013.

[3] J. T. Lin, D. L. Saunders, and S. R. Meshnick, "The role of submicroscopic parasitemia in malaria transmission: what is the evidence?" Trends in Parasitology, vol. 30, no. 4, pp. 183-190, 2014.

[4] F. P. Mockenhaupt, G. Bedu-Addo, C. von Gaertner et al., "Detection and clinical manifestation of placental malaria in southern Ghana," Malaria Journal, vol. 5, article 119, 2006.

[5] S. Omer, E. Khalil, H. Ali, and A. Sharief, "Submicroscopic and multiple plasmodium falciparum infections in pregnant Sudanese women," North American Journal of Medical Sciences, vol. 3, no. 3, pp. 137-141, 2011.

[6] N. O. Wilson, F. K. Ceesay, S. A. Obed et al., "Intermittent preventive treatment with sulfadoxine-pyrimethamine against malaria and anemia in pregnant women," American Journal of Tropical Medicine and Hygiene, vol. 85, no. 1, pp. 12-21, 2011.

[7] O. O. Asa, A. A. Onayade, A. O. Fatusi, K. T. Ijadunola, and T. C. Abiona, "Efficacy of intermittent preventive treatment of malaria with sulphadoxine-pyrimethamine in preventing Anaemia in pregnancy among Nigerian women," Maternal and Child Health Journal, vol. 12, no. 6, pp. 692-698, 2008.

[8] S. Gies, S. O. Coulibaly, F. T. Ouattara, and U. D'Alessandro, "Individual efficacy of intermittent preventive treatment with sulfadoxine-pyrimethamine in primi- and secundigravidae in rural Burkina Faso: impact on parasitaemia, anaemia and birth weight," Tropical Medicine and International Health, vol. 14, no. 2, pp. 174-182, 2009.

[9] R. Afoakwah, J. N. Boampong, A. Egyir-Yawson, E. K. Nwaefuna, O. N. Verner, and K. K. Asare, "High prevalence of PfCRT K76T mutation in Plasmodium falciparum isolates in Ghana," Acta Tropica, vol. 136, no. 1, pp. 32-36, 2014.

[10] Central Region, http://www.wikipedia.org/wiki/Central_Region.

[11] Central Region, http://www.ghanahealthservice.org/ghs-region .php? ghs\&ghsrid $=5$.

[12] H. Zhang and H. Stern, "Sample size calculation for finding unseen species," Bayesian Analysis, vol. 4, no. 4, pp. 763-792, 2009.

[13] M. Cheesbrough, District Laboratory Practice in Tropical Countries (Part 1), Cambridge University Press, 2005.

[14] S. Bereczky, A. Mårtensson, J. P. Gil, and A. Färnert, "Short report: rapid DNA extraction from archive blood spots on filter paper for genotyping of Plasmodium falciparum," The American Journal of Tropical Medicine and Hygiene, vol. 72, no. 3, pp. 249251, 2005.

[15] H. Beck and S. C. Ley, "Monitoring of malaria drug resistance associated SNPs in Plasmodium falciparum on microarray," in Methods in Malaria Research, K. Mell, I. Ljungstrom, H. Perlmann, A. Scherf, and M. Wahlgren, Eds., pp. 248-257, MR4/ATCC, Manassas, Va, USA, 2008.

[16] J. K. Anchang-Kimbi, E. A. Achidi, T. O. Apinjoh et al., "Antenatal care visit attendance, intermittent preventive treatment during pregnancy (IPTp) and malaria parasitaemia at delivery," Malaria Journal, vol. 13, no. 1, article 162, 2014.

[17] A. Danso-Appiah, W. A. Stolk, K. M. Bosompem et al., "Health seeking behaviour and utilization of health facilities for schistosomiasis-related symptoms in Ghana," PLoS Neglected Tropical Diseases, vol. 4, no. 11, article e867, 2010.

[18] L. C. Okell, A. C. Ghani, E. Lyons, and C. J. Drakeley, "Submicroscopic infection in Plasmodium falciparum-endemic populations: a systematic review and meta-analysis," The Journal of Infectious Diseases, vol. 200, no. 10, pp. 1509-1517, 2009.

[19] F. P. Mockenhaupt, B. Rong, H. Till et al., "Submicroscopic Plasmodium falciparum infections in pregnancy in Ghana," Tropical Medicine \& International Health, vol. 5, no. 3, pp. 167173, 2000.

[20] A. A. Adegnika, J. J. Verweij, S. T. Agnandji et al., "Microscopic and sub-microscopic Plasmodium falciparum infection, but not inflammation caused by infection, is associated with low birth weight," American Journal of Tropical Medicine and Hygiene, vol. 75, no. 5, pp. 798-803, 2006.

[21] R. McGready, B. B. Davison, K. Stepniewska et al., "The effects of Plasmodium falciparum and $P$. vivax infections on placental histopathology in an area of low malaria transmission," American Journal of Tropical Medicine and Hygiene, vol. 70, no. 4, pp. 398-407, 2004.

[22] L. C. Okell, T. Bousema, J. T. Griffin, A. L. Ouédraogo, A. C. Ghani, and C. J. Drakeley, "Factors determining the occurrence of submicroscopic malaria infections and their relevance for control," Nature Communications, vol. 3, article 1237, 2012.

[23] A. H. Mohammed, M. M. Salih, E. M. Elhassan et al., "Submicroscopic Plasmodium falciparum malaria and low birth weight in an area of unstable malaria transmission in Central Sudan," Malaria Journal, vol. 12, article 172, 2013.

[24] C. O. Falade, B. O. Yusuf, F. F. Fadero, O. A. Mokuolu, D. H. Hamer, and L. A. Salako, "Intermittent preventive treatment with sulphadoxine-pyrimethamine is effective in preventing maternal and placental malaria in Ibadan, south-western Nigeria," Malaria Journal, vol. 6, article 88, 2007.

[25] L. Hommerich, C. von Oertzen, G. Bedu-Addo et al., "Decline of placental malaria in southern Ghana after the implementation of intermittent preventive treatment in pregnancy," Malaria Journal, vol. 6, article 144, 2007.

[26] M. Luntamo, A.-M. Rantala, S. R. Meshnick et al., "The effect of monthly sulfadoxine-pyrimethamine, alone or with azithromycin, on PCR-diagnosed malaria at delivery: a randomized controlled trial," PLOS ONE, vol. 7, no. 7, Article ID e41123, 2012.

[27] C. Menéndez, A. Bardají, B. Sigauque et al., "Malaria prevention with IPTp during pregnancy reduces neonatal mortality," PLoS ONE, vol. 5, no. 2, Article ID e9438, 2010.

[28] J. Hill, J. Hoyt, A. M. van Eijk et al., "Factors affecting the delivery, access, and use of interventions to prevent malaria in pregnancy in sub-saharan africa: a systematic review and metaanalysis," PLoS Medicine, vol. 10, no. 7, Article ID e1001488, 2013.

[29] S. Thiam, V. Kimotho, and P. Gatonga, "Why are IPTp coverage targets so elusive in sub-Saharan Africa? A systematic review of health system barriers," Malaria Journal, vol. 12, no. 1, article 353, 2013. 

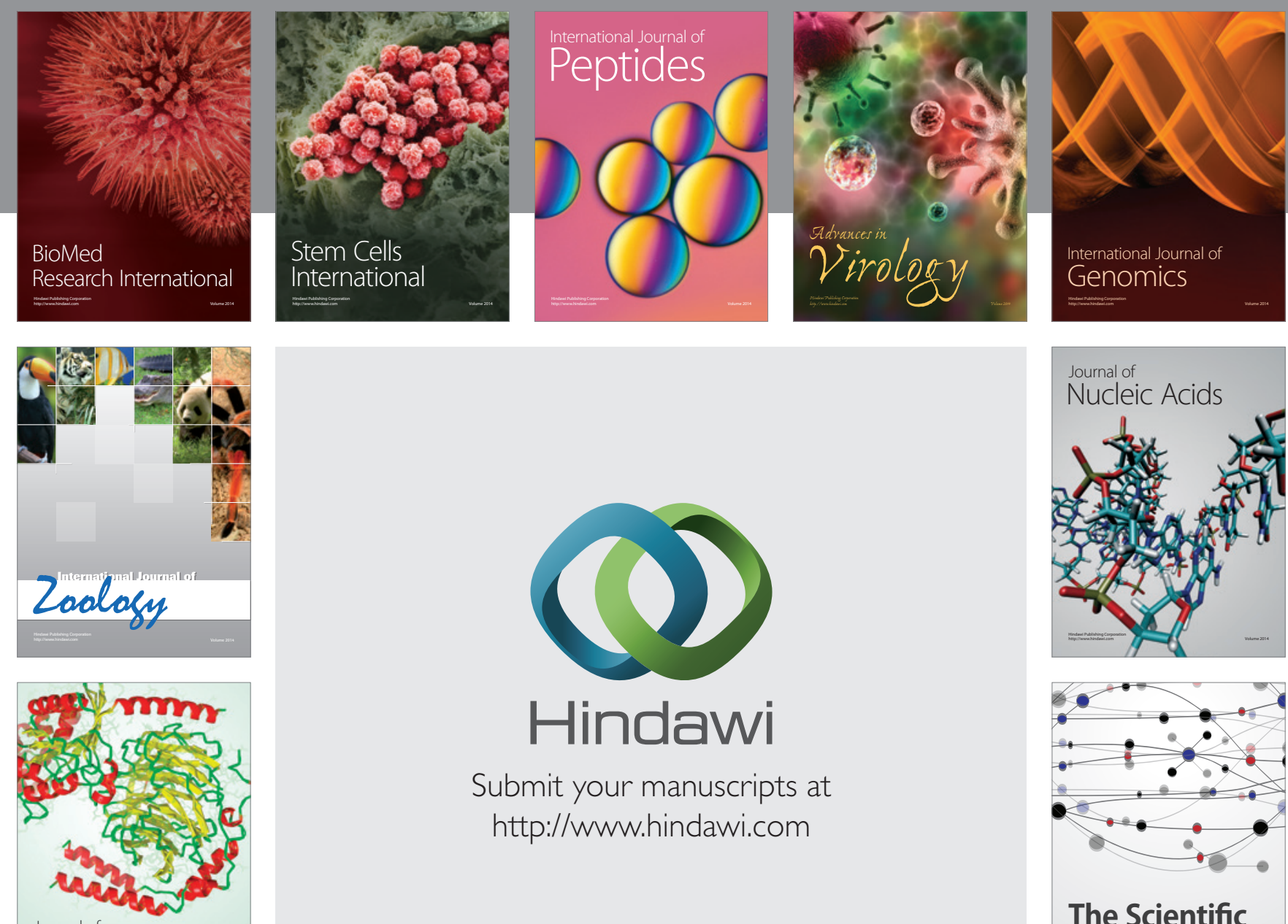

Submit your manuscripts at

http://www.hindawi.com

Journal of
Signal Transduction
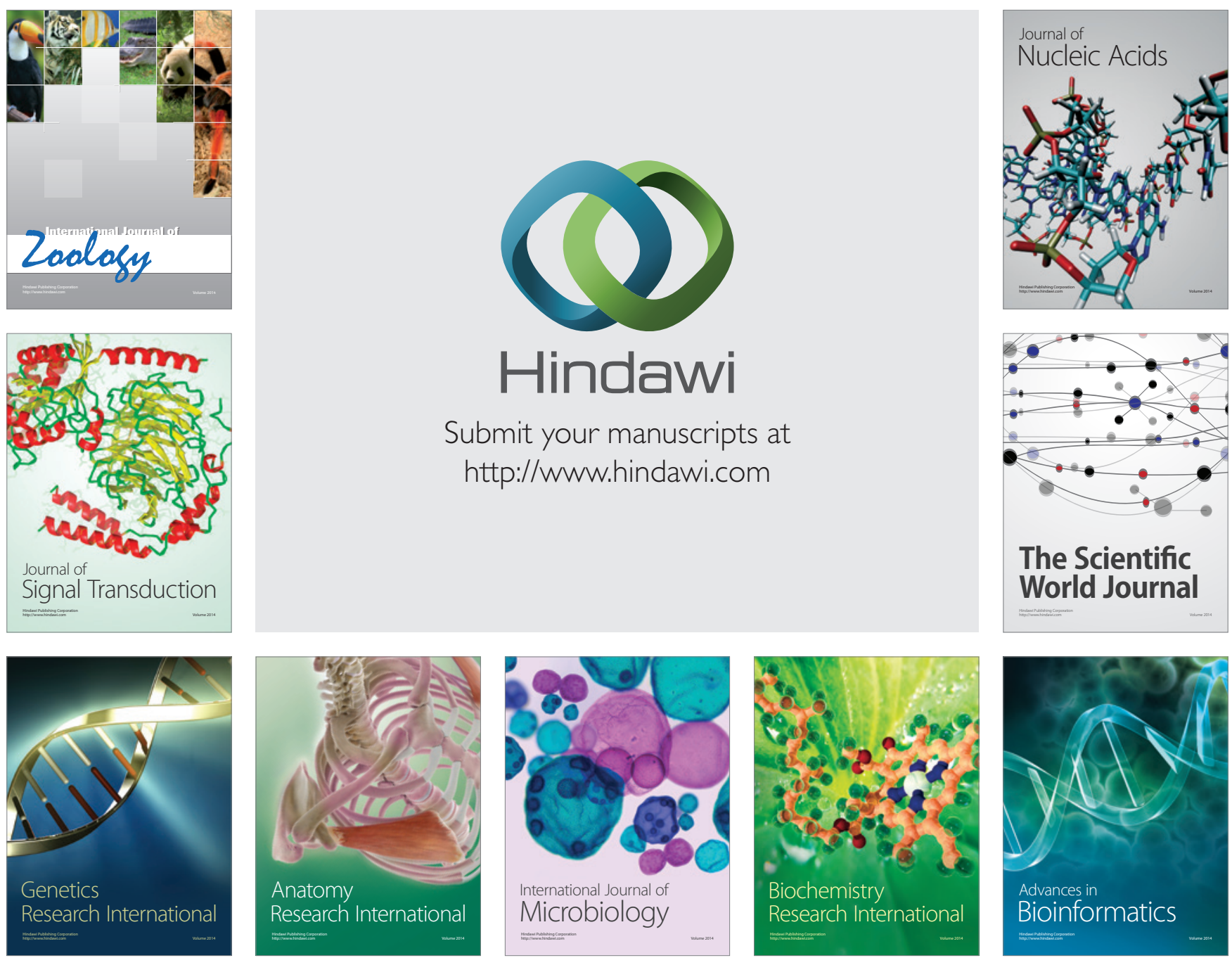

The Scientific World Journal
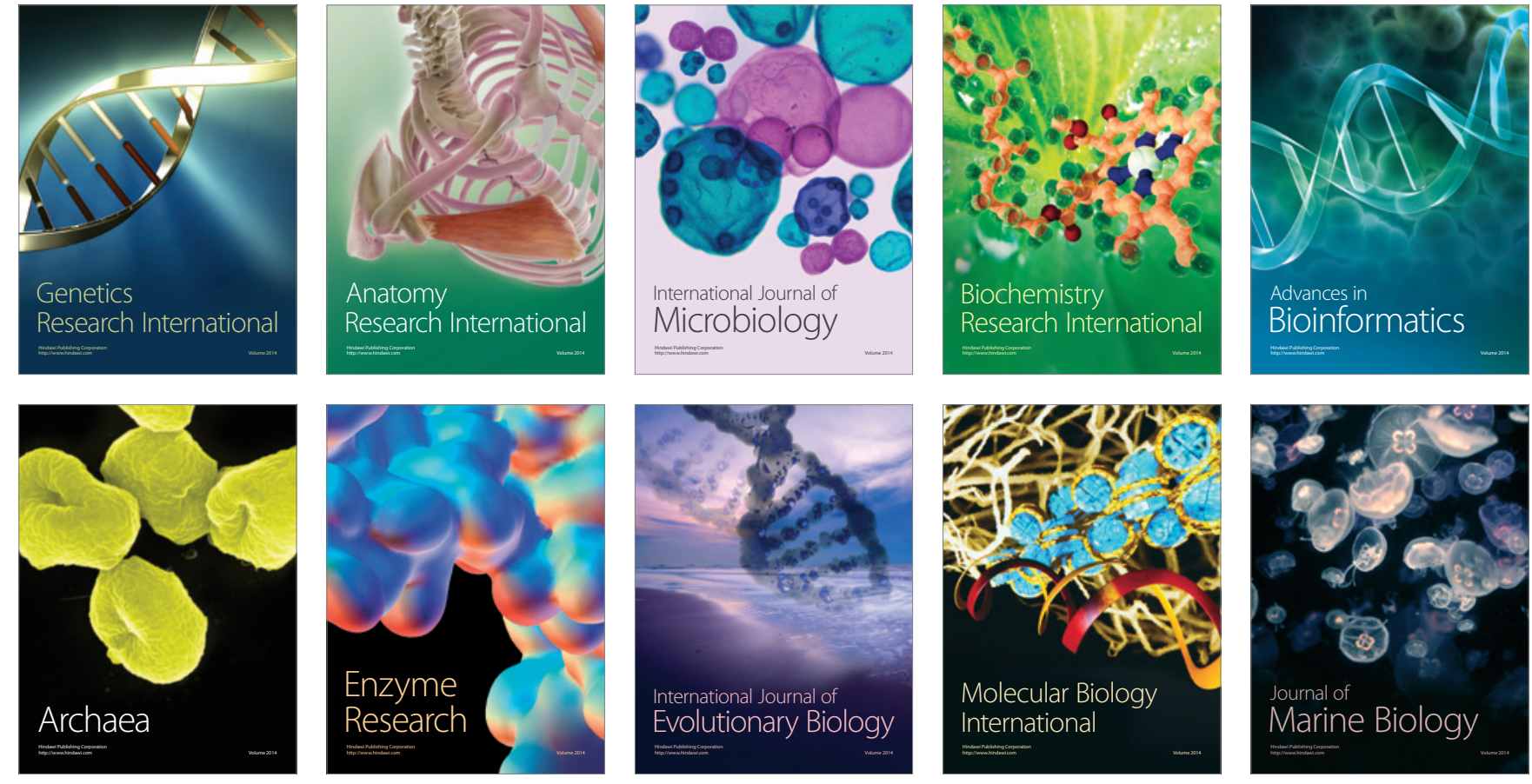